\title{
Why satellite localization beacons are not adapted for marine turtles' study: a sea wireless sensors network solution
}

\author{
Murad Nour Mohammad ${ }^{1}$, Guegan Loic ${ }^{1,2}$, Bonhommeau Sylvain ${ }^{3}$
}

${ }^{1}$ Univ La Reunion, LE2P, St Pierre, Reunion Island, France.

2 Univ Rennes 1, Dept Network \& Telecommun, Technol Univ Inst IUT, St Pierre, Reunion Island, France.

${ }^{3}$ French Res Inst Exploitat Sea, Le Port, Reunion Island, France.

Corresponding authors : email addresses : nour.murad@univ-reunion.fr ;

16000511@etudiant.univ-rennes1.fr ; sylvain.bonhommeau@ifremer.fr

\begin{abstract}
:
This article shows the effects of various parameters like modulation or the radio channel conditions on sea turtles trajectory estimated by terrestrial localization algorithms. This work is part of wireless sensors networks domain in the marine and terrestrial environment in the world of living beings. It allows to identify and understand the parameters that lead to inaccuracies over the sea turtles trajectory. Another important part of this project is to have a better understand about the morphology of the sea turtles and his environment. It also proposes another way to localize sea turtles.
\end{abstract}




\section{INTRODUCTION}

Assessing functional habitats (feeding and reproduction areas) for marine species is critical for conservation and management purposes. Since the 1990s, the development of tags that records the positions of marine species has enabled scientist to better determine these habitats. These devices have been developed to track sea turtles which are currently on the list of endangered species by the International Union for Conservation of Nature (IUCN). There is hence critical needs for conservation measures and better scientific knowledge about these species' ecology.

There are different positioning system modules for the localization of sea turtles available on the market. But they are most often expensive which limits the number of animals that can be tracked and hence the statistical robustness of results. Moreover, researchers have no access to the algorithms used to estimate animal positions. Recent advance in the data transfer for "Internet of Things" applications open new avenues for using this technology in marine application. This technology also offers ways to geolocate objects using algorithms based on the emission power or time from the gateways and transceivers. To overcome the disadvantages of current devices tracking animals, several radio modules are chosen to transfer data to a fixed array of ground stations called gateways based on the LoRa technology [1]. We test different localization algorithms [2] that involve best turtles position estimation and optimal gateways position on the coast or earth. The distances between sea turtles and gateways (GW) will be in the order of kilometers, which is acceptable for this type of localization. Juvenile turtles that we focus the study on are generally foraging in coastal areas.
In order to better understand or apprehend errors on the estimation of the trajectory of the turtles, it is essential to know first of all the biology of the animal and then in a second time to model and to correctly integrate the physical parameters of the channel radio transmission [3]. Thus, from the biological perspective sea turtles swim at different speeds. They may spend very short times at the surface to breath in the millisecond range and more longer times around two or three minutes [4]. In addition from the sea turtle environment perspective, it is necessary to take into account the radio conditions of the over sea environment, which may be extreme in some cases [5], [6]. Wind, waves and sea spray are factors that can alter the signal. This is why we have decided to integrate the over sea radio channel conditions, in order to adapt the algorithms used to attain the best possible accuracy on turtle localization.

Thus, our contribution consists in:

- Design a robust radio transmission against the sea meteorological condition in choosing a robust modulation

- Design a reliable transmitter module for sea turtle

- Show the impact of the number of gateway and their distribution on the trajectory accuracy

- Improve the accuracy of sea turtles trajectories estimation using a better knowledge about the marine radio channel

The rest of the paper is structured as follows. In section III an introduction to sea biology turtle and its behavior are done in order to define constraints on the beacon design. Next the section IV presents the localisation algorithms and the one used in this paper. The section $\mathrm{V}$ gives wireless network topology and the protocol communication. Section VI shows and analyses the impact of some natural and technical parameters on the estimation of sea turtle trajectory. The section VII presents existing localisation technologies and their limits.Finally the section VIII conclude the paper.

\section{ACRONYMS AND NOTATIONS}

- BT: Beacon Turtle

- DGPS: Differential GPS

- GPS: Global Positioning System

- GW: Gateway

- PPS: Precise Positioning System 
- RSSI: Received signal strength indication

- SPS: Standard positioning system

- $d_{i}$ : distance between the beacon turtle to the $i^{t h}$ gateway

- $n_{g}$ : number of gateway

- $p_{t}$ : beacon transmitter power

- $p_{r}$ : gateway receiver power

- $n_{p}$ : size of the preamble field

- $n_{d}$ : size of the data payload field

- $n_{i d}:$ size of the turtle identification field

- $n_{s}$ : size of the stamp field

- $n_{f}$ : size of the beacon message

- $T_{b}$ : bit time

- $T_{f}$ : frame time

- $T_{p i}$ : propagation time for the $i^{\text {th }}$ path

- $\eta$ : gateway receiver radio sensibility

- $D_{\max }:$ maximum distance for a given $\left(p_{t}, \eta\right)$

\section{SEA tURTLE Biology AND CONSTRAins ON THE BEACON DESIGN}

The studied species (green sea turtle, Chelonia mydas) can reach $1.5 \mathrm{~m}$ (carapace length) and weigh up to $400 \mathrm{~kg}$. They live in the tropical and subtropical oceans worldwide. Green sea turtles move across three habitat types, depending on their life stage. They lay eggs on beaches. Mature turtles spend most of their time in shallow, coastal waters with lush seagrass beds. Adults frequent inshore bays, lagoons and shoals with lush seagrass meadows. Entire generations often migrate between one pair of feeding and nesting areas. Within their geographical range, the green sea turtles generally stay near continental and island coastlines. Near the coastlines, the green sea turtles live within shallow bays and protected shores. In these protected shores and bays, the green sea turtle habitats include coral reefs, salt marshes, and nearshore seagrass beds. For these reasons, the size and weight of the beacon is not a strong constrains as it shouldn't be more than $5 \%$ of the animal weight.

Green sea turtles migrate long distances between feeding sites and nesting sites; some swim more than $2600 \mathrm{~km}$ to reach their spawning grounds. Mature turtles often return to the exact beach from which they hatched. Females usually mate every two to four years. In the tropics, green turtles nest throughout the year, although some subpopulations prefer particular times of the year.

Turtles spend most of their first five years in convergence zones within the bare open ocean that surround them. These young turtles are rarely seen as they swim in deep, pelagic waters. Green sea turtles typically swim from 2.5 to $3 \mathrm{~km} / \mathrm{h}$.

Sea turtles spend almost all their lives submerged, but must breathe air for the oxygen needed to meet the demands of vigorous activity. With a single explosive exhalation and rapid inhalation, sea turtles can quickly replace the air in their lungs from 100 to $200 \mathrm{~ms}$. During routine activity, green turtles dive for about 4 to 5 minutes, and surface to breathe for one to three seconds. They can also stay at the surface for several minutes to catch sun and rest. The maximum depth of turtle dives is $200 \mathrm{~m}$ which implies that the beacons should resists to pressure of 20 bar.

Thus multiple constraints should be taken into account. Firstly, the two transmission's options are, during the turtle's surfacing or when the turtle is on the beaches. During the turtle's surfacing, the transmission's time can be short $(100 \mathrm{~ms}$ and $200 \mathrm{~ms}$ ) or long ( $2 \mathrm{~min}$ to $3 \mathrm{~min}$ ) [4]. Moreover in the water, several conditions may affect the transmission's quality such as sea spray, and swell (can be of several meters). On land, sea turtle can be covered by the vegetations and the localization's precision may be inaccuracy or in the worst case impossible. Another constraint called Doppler shift, due to the sea turtle's movements and its environment, should be considered. In fact, this phenomenon causes frequency shift and multipath presence during signal's transmission. All of these lead to a bad reception on GW.

\section{LOCALIZATION TECHNIQUES OVERVIEW}

Various beacon emitter localization techniques are possible, each of them has specific advantages and disadvantages. Generally, emitter localization techniques are based upon one or more measure of one or more of the following properties :

- The received signal power (Received Signal Strength, Power of Arrival) [7] [8]

- The signal's propagation time (Time Of Arrival, Time Difference Of Arrival) [2] [9] [10]

- The direction of arrival (Angle Of Arrival) [2]

- Relative shifts in the signal frequency observed at pairs of spatially separated sensors, at least one of which is moving (Frequency Difference Of Arrival) [11]

The main emitter localization technique applied here uses measurement of the received signal strength (RSS) obtained from the individual GW, the GW position and a path loss model that relates the path loss to the propagation distance. A particular simple case occurs for free space propagation. Here the path loss of an electromagnetic wave is proportional to $1 / d_{i}^{2}$. Therefore, by knowing the BT transmitter power $p_{t}$, the distance between the BT and GW can be determined from a properly calibrated measure of the $\mathrm{GW}$ received signal power $p_{r}$. This distance directly defines the equation of a line corresponding to the radius of a circle centered on the GW position. Each point of this circle defines a possible position of the BT. If two GW are used, the result will be a pair of circles that normally intersect at two points, one of which corresponds to the BT location. Consequently, using $n_{g} \mathrm{GW}$ (with $n_{g}>2$ ), the BT position will correspond to a cluster of $n_{g} \times\left(n_{g}-1\right) / 2$ intersections. Of course, this approach implies an ideal over sea radio channel. Furthermore, in a marine environment, the received signal power $p_{r}$ will depend on various factors, such as the heights of the transmission and reception antennas and the nature of the sea. The sea effects are particularly important. In practice the path loss will not be a monotonic function of the distance and the underlying dependence on distance can vary between $1 / r^{2}$ and $1 / r^{4}$. To be able to approximate the distance between the BT and the GW using POA technique, the RSSI of a 
receive signal transmitted by BT need to be measured on GW. This metric is measured with a small inaccuracy according to the Radio Frequency (RF) module on GW. Next with the strong hypothesis the use of a path loss channel, it's possible to approximate the distance between BT and GW. Friis equation in decibel, known as telecommunication equation is used as the path loss model (see equation 1).

$$
p_{r}\left(d_{i}\right)=20 . \log _{10}\left(\frac{p_{t} \cdot G_{t} \cdot G_{r} \cdot \lambda}{4 \cdot \pi \cdot d_{i}}\right)+30
$$

With :

$d_{i}$ : distance between the BT to the $i^{\text {th }} \mathrm{GW}(\mathrm{m})$

$p_{r}:$ GW received power or RSSI $\left(d B_{m}\right)$

$p_{t}: \mathrm{BT}$ transmitter power $(W)$

$G_{t}:$ transmitter antenna linear gain

$G_{r}$ : receiver antenna linear gain

$\lambda$ : wavelength (meters)

The distance $d_{i}$ can easily be deducted from equation 1 as,

$$
d_{i}=\frac{\lambda}{4 \pi} \times \frac{1}{\sqrt{\frac{1}{P_{t} G_{t} G_{r}} \times 10^{\frac{P_{r}-30}{10}}}}
$$

So classical technique to locate a beacon's signal origin using the POA technique, is to use multilateration. Multilateration enable to approximate the position of a beacon by knowing the distance that separating this beacon from other knowing positions called anchors. Then with the different distances $d_{i}$ from Beacon Turtle (BT) and each GW $i$, three circles can be drawn if three $\mathrm{GW}$ are used (see figure 1). With the intersections formed by the circles, we can compute line equations and found their intersection to finally approximate the turtle position.



Fig. 1. Multilateration using three gateways.

In some cases, two circle intersections can coincide and is considered as one point. Therefore no intersection lines can be drawn because there is an infinite number of line equation that passing thought one point.

Let's consider now three $\mathrm{GW}$. The coordinate of the $i^{t h} \mathrm{GW}$ is the couple $\left(x_{i}, y_{i}\right)$. If the emitter BT is located at $(x, y)$, the distance from the emitter to the $i^{t h} \mathrm{GW}$ is given by

$$
d_{i}=\sqrt{\left(x-x_{i}\right)^{2}+\left(y-y_{i}\right)^{2}}
$$

Finally, we can estimate the position $(x, y)$ of the BT.

\section{WIRELESS NETWORK TOPOLOGY AND COMMUNICATION PROTOCOL}

In order to determined a good network topology and his feasibility, the Europa Island field and the communication protocol need to be described and analyzed. Therefore Europa Island visible in the figure 2 is about $6 \mathrm{~km}$ from west to east and $7 \mathrm{~km}$ from north to south. So a coverage area of $S=15 \mathrm{~km}^{2}$ will be under observation and oversight.

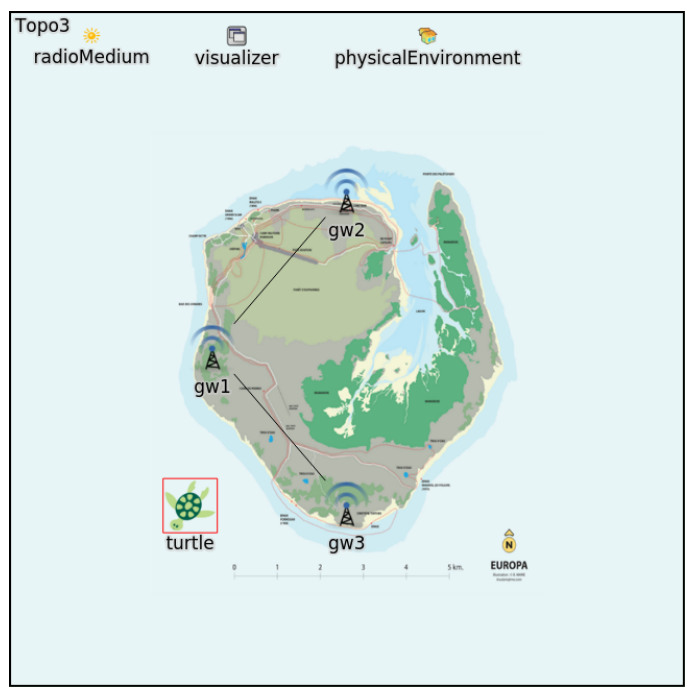

Fig. 2. Europa Island.

When the turtle take a breath, a pressure sensor detects if the turtle is under or above the water. Then, the BT broadcasts a beacon message (figure 3 ). The beacon message contains four fields. First, the preamble field of size $n_{p}=6$ bits is useful to estimate the radio channel's profile $\left(p_{r}, T_{p i}\right)$. It consists of a particular bit sequence with some mathematical properties like equiprobability of zero and one, periodicity, cyclicity and stationarity. The preamble sequence is known from the BT and the GW. The second field contains the turtle identity with a size of $n_{i d}=1$ byte. So a maximum of 256 turtles could be identified with the beacon. The third field called stamp of size $n_{s}=1$ byte allow all $\mathrm{GW}$ to maintain the order of the received frames. And the last field is the data that contain physiological parameters and the transmission time for TOA with a size of $n_{d}=8$ bytes. Finally, beacon message size is express in the following way $n_{f}=n_{p}+n_{i d}+n_{s}+n_{d}=86$ bits.

Furthermore the electronic beacon don't encode the data and stays simple in order to save energy and to send the message efficiently. At a given GW receiver, all fields of the message 


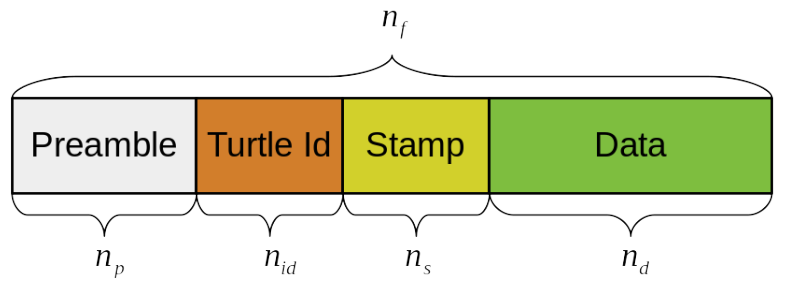

Fig. 3. Frame structure of the beacon message.

are demodulated except the preamble field (see figure 4). This last is correlated with the original sequence preamble known by the BT and a given GW. The correlator function estimates the power and the delay profile of the sea radio channel instantaneously. Then we could estimate the received power $p_{r}$ and the propagation's delay $T_{p}$ of the main path shown in figure 5. In order to highlight the main path we represent the radio channel's profile with its normalized power [3]. To finish, the couple $\left(p_{r}, T_{p i}\right)$ is sent to the POA localization algorithm. This last estimates and saves the position of the BT on a data base.

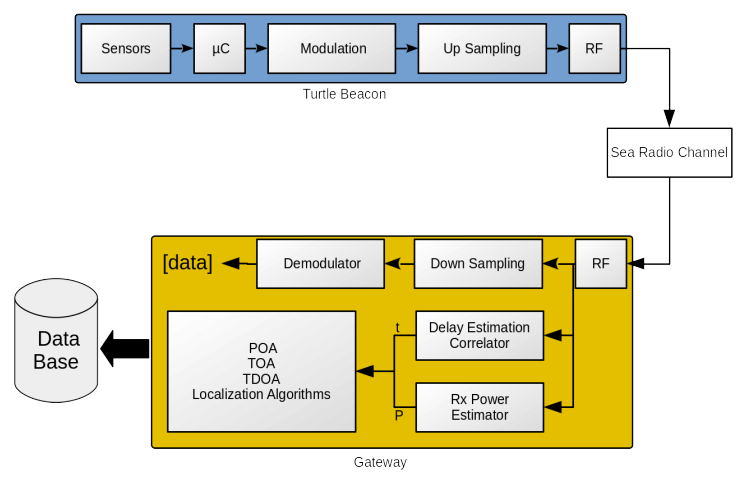

Fig. 4. Link transmission between turtle beacon and a gateway.

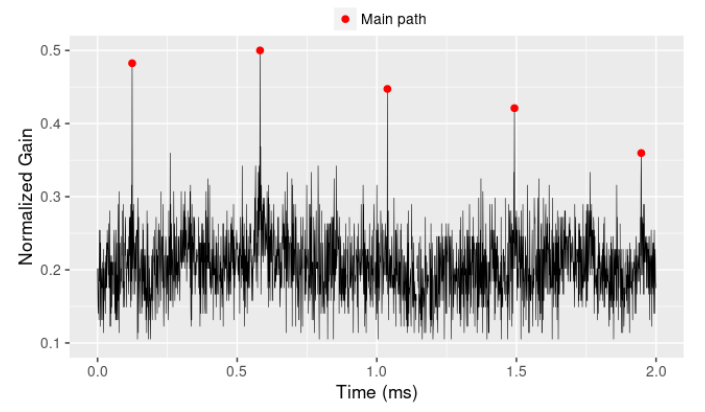

Fig. 5. Normalized power and delay estimation sea radio channel profile.

Another question to deal with, is the sending frequency of the beacon message. As the bit rate $D_{b}=37.5 \mathrm{kbps}$, the frame duration is equal to $T_{f}=231.424 \mathrm{~ms}$. So during the short breaths of the turtle the BT transmitter could transmit 1 frame and during the long breath it could transmit 648 frames. The fact that the beacon message is sent frequently could improve the position estimation.

\section{RESUlts AND ANALYSis}

Simulation parameters are given in table I. All parameters computed in this table suppose that we are using the SX1276 Lora RF module [12]. In order to compare all results, we use the same known trajectory (see figure 6) of the turtle for each condition factor like the radio channel's conditions, the modulation, the number of $\mathrm{GW}$, the position of $\mathrm{GW}$ and the GW distribution.

TABLE I

SIMULATION PARAMETERS.

\begin{tabular}{|c||c|}
\hline Parameters & Value \\
\hline$n_{g}$ & variable (3 by default) \\
\hline \hline$P_{t}$ & $13 \mathrm{dBm}(20 \mathrm{~mW})$ \\
\hline \hline$n_{p}$ & 6 bits \\
\hline \hline$n_{i d}$ & 8 bits \\
\hline \hline$n_{f}$ & 86 bits \\
\hline \hline$T_{b}$ & $2.69 \mathrm{~ms}$ \\
\hline \hline$\eta$ & $-148 \mathrm{dBm}$ \\
\hline \hline$T_{f}$ & $231.4 \mathrm{~ms}$ \\
\hline
\end{tabular}

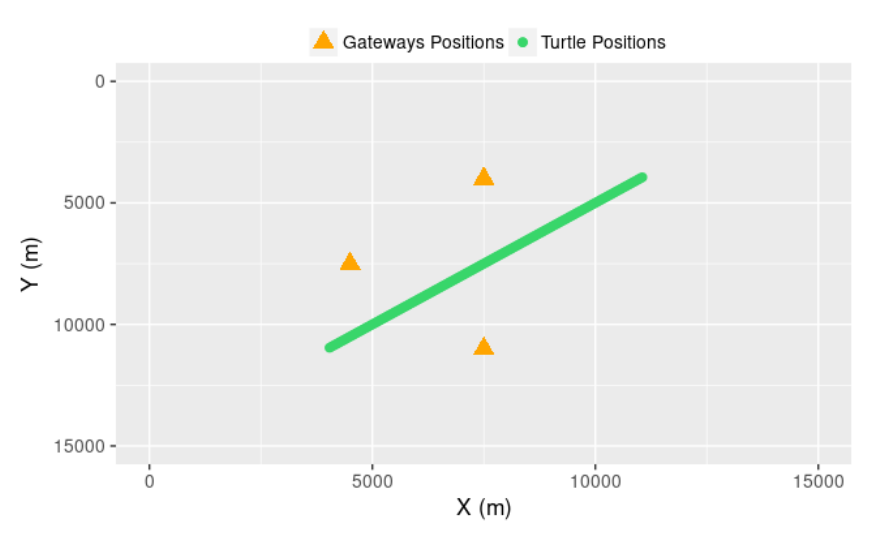

Fig. 6. A arbitrary turtle linear trajectory.

\section{A. Radio Channel's Impact}

The radio channel has a direct impact on the turtle trajectory estimation. In fact, depending on the sea radio conditions, the GW may be unable to receive the signal from the turtle beacon and data loss can occurs (see figure 7). We define two realist path loss channels based on actual measurement. This two channels are visible on table II. To build this two channels, we place a turtle beacon on the ground and a GW transmitter at a distance of $20 \mathrm{~m}$. Channel 1 was obtained by placing the GW at 1 meter of the ground and channel 2 was obtained by placing it directly on the ground. It must be noted that, the GW height need to be taken into account in the parameters of the reception's quality. The analysis of the table III compares the two realistic channels to an ideal channel and its shows that the radio channel's conditions have lot of impact on the sea turtle's localization accuracy. Tiny variations of the radio channel's conditions may cause bad reception of the signal by the GW. 
TABLE II

TWO REALISTIC RADIO CHANNELS

\begin{tabular}{|c|c|c|c|c|}
\cline { 2 - 5 } \multicolumn{1}{c|}{} & \multicolumn{2}{c|}{ Channel 1 } & \multicolumn{2}{c|}{ Channel 2 } \\
\hline Paths & Delays $(\mu \mathrm{s})$ & Gain $(\mathrm{dB})$ & Delays $(\mu \mathrm{s})$ & Gain $(\mathrm{dB})$ \\
\hline 1 & 0 & -0.38 & 0 & -3.69 \\
\hline 2 & 18.38 & -5.0 & 11.73 & -5.36 \\
\hline 3 & 27.19 & -4.74 & 26.89 & -5.14 \\
\hline
\end{tabular}
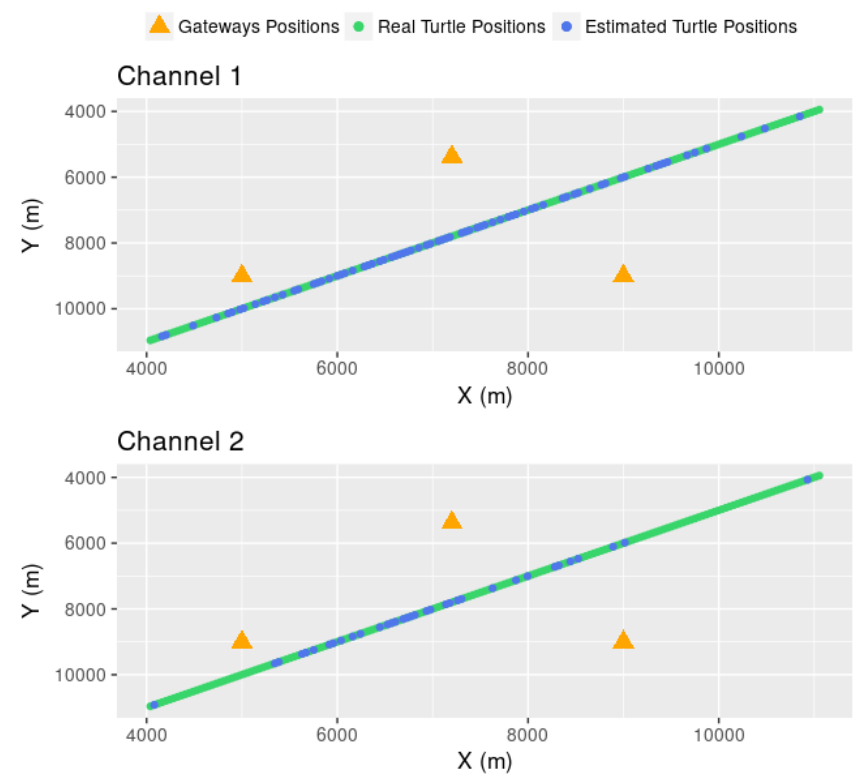

Fig. 7. Impact of the radio channel.

TABLE III

ANALYSIS OF THE RADIO CHANNEL IMPACT

\begin{tabular}{|c|c|c|c|c|}
\cline { 2 - 5 } \multicolumn{1}{c|}{} & Total & Found & Lost & Error Rate \\
\hline Ideal & 173 & 173 & 0 & $0 \%$ \\
\hline Channel 1 & 173 & 68 & 105 & $60 \%$ \\
\hline Channel 2 & 173 & 39 & 134 & $77 \%$ \\
\hline
\end{tabular}

\section{B. Modulations Impact}

The modulation reliability to the radio channel conditions is shown in figure 8 . This figure shows three modulations that are part of the Lora SX1276 module [12]. According to the modulation's plots, frequency-shift keying (FSK) is more robust compared to phase modulation like minimum-shift keying (MSK) and gaussian minimum-shift keying (GMSK). In fact these phase modulations are more subject to multi path which delay the signal's phase. For example, with $S N R=0 \mathrm{~dB}$ the $B E R \approx 2 \%$ with the FSK modulation against $B E R \approx 15 \%$ for GMSK and $B E R \approx 25 \%$ for MSK.

\section{Gateway number impact}

The figure 9 shows the effect of the number of gateway on turtle trajectory errors. The more $n_{g}$ increases the less error of trajectory occurs. So with $10 \mathrm{GW}$, we have an error of trajectory around $4 \%$. However in order to maintain a suitable balance between environment and localization accuracy, we decide to use $8 \mathrm{GW}$ that corresponding to a trajectory error

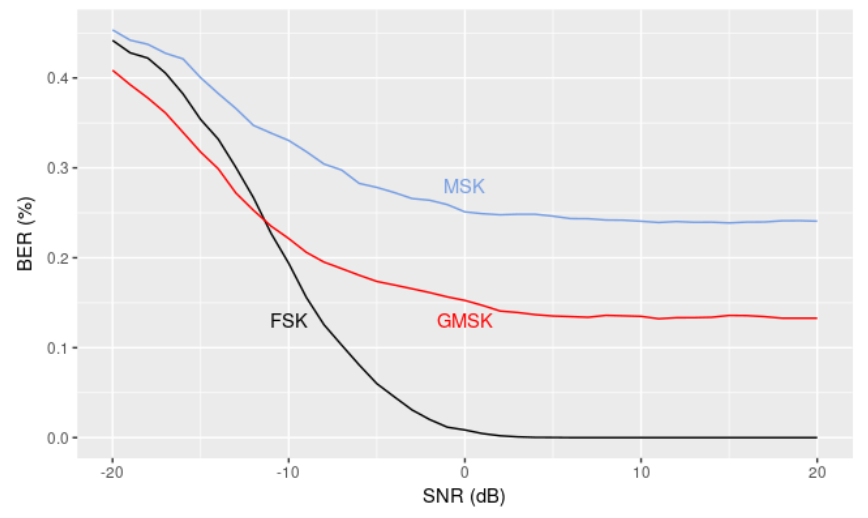

Fig. 8. Impact of the modulation choice.

around $10 \%$. The real trajectory consists of 173 known points scattered over a distance of $10 \mathrm{~km}$ traveled by the turtle. With $8 \mathrm{GW}, 156$ points are found against 17 points lost. So a compromise between the respect of the environment [13], the GW power supply limited by batteries and a reasonable error rate was found.

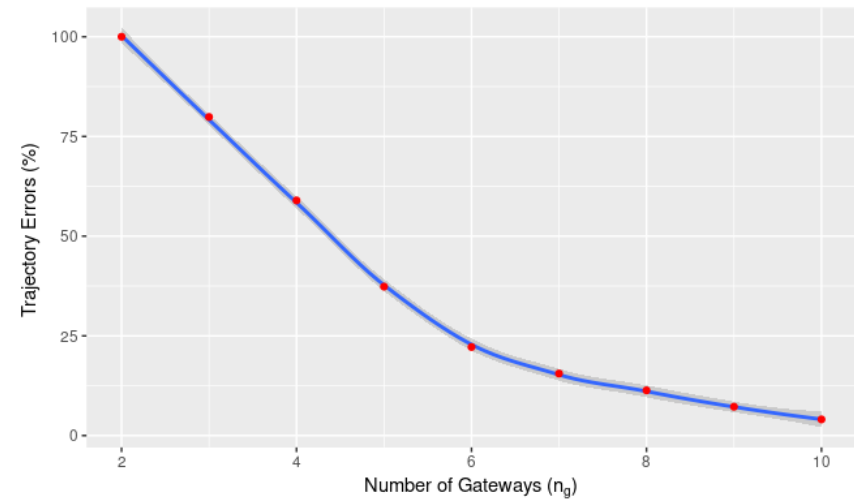

Fig. 9. Impact of gateway number.

\section{Gateway disposition impact}

The GW are disposed at equal distances $D_{\max }$, to obtain the best radio signal coverage. The different way to distribute the GW can significantly decrease the number of position errors and improve the localization accuracy. The influence of the GW geometrical distribution are shown in table IV. Disposed the GW linearly, led to a position error of $100 \%$. In fact, all lines built from circle intersections will be vertical, and no line intersections will be possible. So, no conclusion can be made about the turtle's position. According to the table IV, the best gateway distribution from the available patterns is the hexagonal pattern.

\section{RELATED WORK}

We introduce here some work based on existing techniques that can be used to find sea turtles' position [14] living in different part of the world like in South Pacific Ocean [15] 
TABLE IV

IMPACT OF THE GATEWAYS GEOMETRICAL DISTRIBUTION.

\begin{tabular}{|c||c||c|}
\hline Disposition & $n_{g}$ & \% Position error \\
\hline Linear (aligned) & 3 & 100 \\
\hline \hline Equilateral triangle & 3 & 84.12 \\
\hline \hline Arbitrary triangle & 3 & 88.81 \\
\hline \hline Square & 4 & 63.41 \\
\hline \hline Hexagonal pattern & 6 & 28.34 \\
\hline
\end{tabular}

or in Indian Ocean [16]. This section deals with the state of play of the beacons used on animals to locate or collect some physiological data.

Argos is a beacon [17] which transmits at a carrier frequency of $401.650 \mathrm{MHz}$, with a power of $250 \mathrm{~mW}$ and a variable transmission rate between $40 \mathrm{~s}$ and $200 \mathrm{~s}$ with a step of 10s. The transmission duration varying from 360 to $920 \mathrm{~ms}$ according to the beacon message length (from 32 bits to 256 bits). Three NOAA satellites in polar orbits ensures the Argos beacons localization. On circular orbits at $850 \mathrm{~km}$ the satellite can receive waves transmitted by all beacons in a circle of $5000 \mathrm{~km}$ of diameter on the Earth's surface. According to the beacon latitude, the satellite passing 7 times a day on the equator and 28 times on the poles [18]. The beacon localization are made in the following manner. When the satellite is passing over the beacon, it receives a signal on a specific frequency, code and repetition. In other words, all beacons transmit at the same frequency, but with different code and at different times. The measure of Doppler Shift, and the use of particular algorithms gives the beacon position. The given position by this technique has an accuracy in a range of 150 to $300 \mathrm{~m}$ [19].

These Argos beacons are limited by their prices around $5000 €$, their lifetimes ( 1 year), they need to reach a maximum of $5 \%$ of the animal's weight but also a limited amount of recorded data around 356 bits. Moreover, the Argos beacon's communication is very expensive. Unlike the Argos technology, the Lora beacon is able to transmit with a power of $20 \mathrm{~mW}$ at carrier frequency of $868 \mathrm{MHz}$. This particularity of the Lora module allows to increase its operating time. Moreover, a sea turtle Lora transmitter is much less expensive (around $300 €$ ) than an Argos beacon and the communication band is free of charge.

The LOng RAnge Navigation system (LORAN) [20] is a radio navigation system that use the fixed terrestrial emitter waves to establish a position. The LORAN is a hyperbolic type system. Hyperbolic systems determine the position by measuring the difference in propagation time between a minimum of two transmitters and the points' positions with equal difference is an hyperbola on the map. Three transmitters are required for hyperbolas' intersection point. The first hyperbolic system, LORAN-A, operated at $1800 \mathrm{kHz}$, the LORAN-C operated at $100 \mathrm{kHz}$. The maintenance of LORAN, less accurate and with a limited coverage, is periodically challenged to be replaced by new generation localization beacons like Global Positioning
System (GPS).

There is various localization beacons like the American GPS, the Russian GLONASS, the European Galileo or the Chinese Beidou. The GPS works with Precise Positioning System (PPS) [21] in no degraded mode or Standard Positioning System (SPS) [22] in degraded mode. The SPS signal offers to the public an accuracy in a range of $10 \mathrm{~m}$ to $100 \mathrm{~m}$ horizontally. The GPS receiver sensibility range from $-165 \mathrm{dBm}$ in tracking mode to $-148 \mathrm{dBm}$ in acquisition mode [23]. GPS provide some limitations. The signal's transmission quality can be easily altered and the communication is impossible when the sea turtle is below the surface (interrupted localization). Some devices use the «Fastloc » technology to quickly locate the animal's surfacing. These devices record and transfer raw data from GPS satellites. Once the data gathered, the animal position can be estimated with calendars and ephemerids of satellites by using algorithms. This technique provides a precision up to $70 \mathrm{~m}$ [24]. GPS beacons are more accurate than our localization technique. In fact the Lora's localization technique provide a precision around $50 \mathrm{~m}$. But GPS is a passive beacon and no physiological's data can be transmitted. Moreover GPS and Fastloc positions need to be collected and they are not real time systems.

\section{CONCLUSION}

Lot of parameters can alter the localization techniques' accuracy and need to be considered. Firstly the modulation and FSK was selected because we proved that its more robust to the effects of the radio channel conditions. Secondly the number of $\mathrm{GW}$ is another interesting parameter, and a number of 8 GW will be enough. Finally the geometrical distribution of the GW has an impact on the localization's accuracy. So, octagon geometrical distribution of the GW is a correct solution. The next step of our work consist in the development of a beacon and doing field experiments to compare the field measurements to the simulated measurements.

\section{REFERENCES}

[1] Technical Marketing Workgroup 1.0. "A Technical Overview of LoRa and LoRaWAN." Lora Alliance, November 2015.

[2] Frédéric Evennou, "Techniques et Technologies de Localisation Avancées Pour Terminaux Mobiles Dans Les Environnements Indoor," Université Joseph-Fourier-Grenoble I, 2007. https://tel.archivesouvertes.fr/tel-00136064/.

[3] L. Guegan, N. M. Murad, J. M. Lebreton and S. Bonhommeau, "Integrating Over Sea Radio Channel for Sea turtles Localization in the Indian Ocean", IEEE RADIO 2017 Conference, Cape Town South Africa, September 25th-28th 2017, 2 pages (accepted).

[4] Southwood, Amanda L., Russel D. Andrews, Molly E. Lutcavage, Frank V. Paladino, Nigel H. West, Robert H. George, and David R. Jones, "Heart Rates and Diving Behavior of Leatherback Sea Turtles in the Eastern Pacific Ocean.” Journal of Experimental Biology 202.

[5] Huang, Fang, Xuefei Liao, and Yong Bai, "Multipath Channel Model for Radio Propagation over Sea Surface" Wireless Personal Communications 90, no. 1, September 2016, 245-57. doi:10.1007/s11277-016$3343-4$.

[6] Wu, Yanyang, Zhibin Gao, Canbin Chen, Lianfen Huang, Hua-Pei Chiang, Yueh-Min Huang, and Hao Sun, "Ray Tracing Based Wireless Channel Modeling over the Sea Surface near Diaoyu Islands," 124-28. IEEE, 2015. doi:10.1109/CCITSA.2015.35. 
[7] Zhu, Xiuyan, and Yuan Feng. "RSSI-Based Algorithm for Indoor Localization." Communications and Network 05, no. 02 (2013): 37-42. doi:10.4236/cn.2013.52B007.

[8] B.R., Jackson, Wang S., and Inkol R. "Emitter Geolocation Estimation Using Power Difference of Arrival." Canada: Defence R\&D Canada, Mai 2011.

[9] Kaune, Regina. "Accuracy Studies for TDOA and TOA Localization." IEEE. August 31, 2012.

[10] Aatique, Muhammad. "Evaluation of TDOA Techniques for Position Location in CDMA Systems." Virginia Polytechnic Institute and State University, 1997.

[11] Shao, Hongshuo, Dongkyun Kim, and Kwanho You. "TDOA/FDOA Geolocation with Adaptive Extended Kalman Filter." In Grid and Distributed Computing, Control and Automation, 226-35. Springer, 2010.

[12] SEMTECH. "SEMTECH SX1276 Datasheet,"

[13] "Les îles éparses." Terres Australes et Antarctiques Françaises (TAAF), Juillet 2011

[14] M. S. Coyne and B. J. Godley, "Satellite Tracking and Analysis Tool (STAT): an integrated system for archiving, analyzing and mapping animal tracking data”, Marine Ecology Progress Series, Vol; 301, pp.1-7, 2005, doi:10.3354/meps301001

[15] M. C. Boyle, N. N. FitzSimmons, C. J. Limpus, S. Kelez, X. VelezZuazo and M. Waycott, "Evidence for transoceanic migrations by loggerhead sea turtles in the southern Pacific Ocean", Proceeding of the Royal Society B, Biological Sciences, 7 pages, 4 March 2009.DOI: 10.1098/rspb.2008.1931

[16] FEDER Mégafaune Project, " Distribution spatiale des localisations de la Tortue Verte (Chelonia mydas) suivis par balise Argos depuis Tromelin (TAAF)", 2015. http://sextant.ifremer.fr/record/1c8f18db-fdd54c8e-b4eb-ea43ee165389/

[17] http://www.argos-system.org

[18] https://argos-mission.cnes.fr

[19] Michel Peberay, "Présentation des balises Argos", document 4.5, Electronique Marcel Dassault, CEFIGRE, Séminaire télétransmission, France

[20] L. N. Ridenour and all, "LORAN", MIT, Radio Laboratories Series, 1948, Mac Graw-Hill Book Company, first edition, http://www.loran-
history.info/downloads/MIT-Radiation-Lab-Series-V4-Long-RangeNavigation-LORAN.pdf

[21] Department Of Defense. "Global Positioning System Standard Positioning Service Performance Standard," September 2008 http://www.gps.gov/.

[22] Department Of Defense. "Global Positioning System Precise Positioning Service Performance Standard," February 2007. http://www.gps.gov/.

[23] Skylab, "Ultra High Sensibility and Low Power GPS Receiver Module", GPS Module Datasheet, SKG13BL.

[24] Dujon, Antoine M., R. Todd Lindstrom, and Graeme C. Hays. "The Accuracy of Fastloc-GPS Locations and Implications for Animal Tracking." Edited by Patricia Backwell. Methods in Ecology and Evolution 5, no. 11 (November 2014): 1162-69. doi:10.1111/2041-210X.12286. 